\title{
Analysis of dam-reservoir interaction by employing the modified-efficient fluid hyper-element
}

\author{
A. A. $\operatorname{Sani}^{1} \&$ V. $\operatorname{Lotfi}^{2}$ \\ ${ }^{1}$ Fellow of Islamic Azad University of Mashhad, Iran \& Ph.D. Candidate \\ of Civil Engineering Department, Amirkabir University of technology, \\ Tehran, Iran. \\ ${ }^{2}$ Professor of Civil Engineering Department, Amirkabir University of \\ technology, Tehran, Iran.
}

\begin{abstract}
In this paper, a FE-(FE-HE) procedure is implemented for dynamic analysis of concrete arch dams, in the frequency domain. The technique relies on the finite element method for the discretization of the dam body and the near field of the reservoir, while the far field is handled by the fluid hyper-element. It should be emphasized that the usual method for calculating the required impedance matrix of the above mentioned fluid hyper-element is dependent on the solution of a complex eigen-value problem for each frequency, which is very time-consuming. This problem was remedied by an efficient technique in a previous study which greatly reduces the computational time. The present approach is a modification of that procedure trying to have similar efficiency without losing the accuracy in any cases, contrary to the previous study. Furthermore, a previously developed special purpose program is enhanced based on this new method and the response of the Morrow Point arch dam is studied as an example
\end{abstract}

Keywords: dam-reservoir interaction, fluid hyper-element, efficient dynamic analysis.

\section{Introduction}

There are different alternatives for dynamic analysis of concrete dams [1-3]. However, the rigorous analysis of concrete arch dam-reservoir system is based on the FE-(FE-HE) method (i.e., Finite Element-(Finite Element-Hyper Element)). This means, the dam is discretized by solid finite elements, while, the reservoir is divided into two parts, a near field region (usually an irregular shape) in the vicinity of the dam and a far field part (assuming uniform channel), which 
extends to infinity. The former region is discretized by fluid finite elements and the latter part is modeled by a three-dimensional fluid hyper-element. The analysis is carried out in frequency domain either by direct approach [4], or sub-structuring techniques [5, 6]. Regardless of the option selected among these rigorous techniques, a major portion of the numerical calculation time spent is due to the solution of a complex eigen-value problem related to fluid hyperelement, which must be solved at each frequency.

To remedy this, an efficient procedure was proposed in a previous study [7] for the required impedance matrix calculation of the fluid hyper-element, which greatly reduces the computational time. Although, the method was very accurate in most practical conditions, it was estimated that it would introduce errors in the range of $10 \%$ under certain circumstances. It should be noticed that the mentioned results were obtained by employing the special purpose program "MAP-76" which was enhanced for that investigation.

In the present study, a modified efficient procedure is proposed for the abovementioned problem. In fact, the new approach is envisaged to be an enhancement of a previously proposed efficient method [7]. This approach is mainly relying on mode shapes corresponding to an arbitrary frequency. It is also noticeable that the original efficient method can be considered as a special case of the modified efficient procedure.

The applied concepts and the required changes in the formulation can be found elsewhere in detail [8]. Meanwhile, the aforesaid special purpose program is improved based on the proposed theory, and the response of Morrow Point arch dam is obtained for various conditions. This is utilized to investigate the accuracy of the modified efficient procedure thoroughly.

\section{Modelling and basic parameters}

As mentioned, the dam body and the near field region of reservoir in the vicinity of the dam are treated in this work by finite elements. Also, a far field part of reservoir which extends to infinity is modeled by a modified-efficient fluid hyper-element which is proposed in this study.

\subsection{Models}

An idealized symmetric model of Morrow Point arch dam is considered. The geometry of the dam may be found in reference [5].

The dam is discretized by 40 isoparametric 20 -node solid finite elements (fig. 1). The water domain is divided into two regions (fig. 2). The near-field part is considered as a region, which extends to a specified length $\mathrm{L}=0.2 \mathrm{H}$ ( $\mathrm{H}$ being the dam height or maximum water depth in the reservoir), which is measured in upstream direction at dam mid-crest point. The far-field region starts from that point and extends to infinity in the upstream direction. The near-field region is discretized by 80 isoparametric 20 -node fluid finite elements, while the far-field region is modeled by a fluid hyper-element which itself is constructed from 
40 isoparametric 8-node sub-elements. The complete model of dam-reservoir system is shown in fig. 3 .

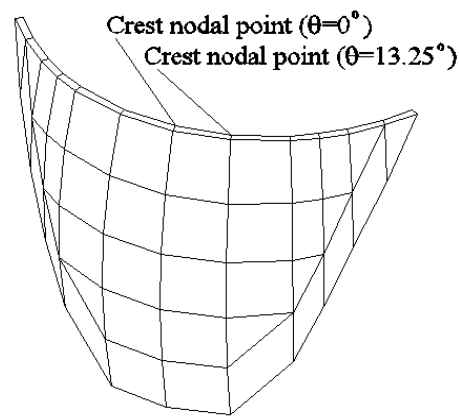

Figure 1: $\quad$ Finite element mesh of the dam body.
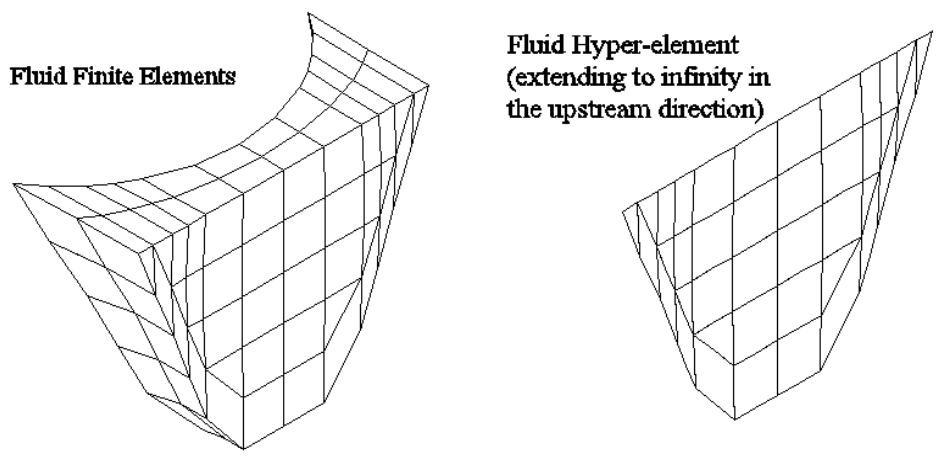

Figure 2: Discretization of water domain (fluid finite elements $(\mathrm{L} / \mathrm{H}=0.2)$, and the fluid hyper-element)

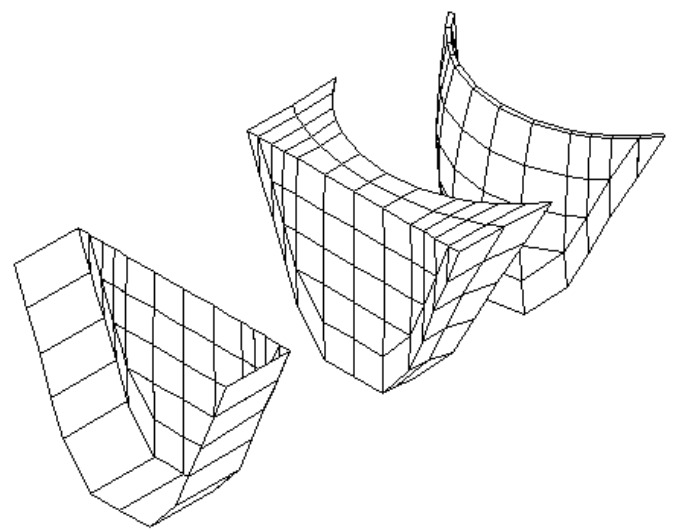

Figure 3: Complete dam-reservoir system: FE-(FE-HE). 


\subsection{Basic parameters}

The dam concrete is assumed to be homogeneous with isotropic linearly viscoelastic behavior and the following main characteristics:

$\begin{array}{ll}\text { Elastic modulus }\left(\mathrm{E}_{\mathrm{d}}\right) & =27.5 \mathrm{GPa} . \\ \text { Poisson's ratio } & =0.2 \\ \text { Unit weight } & =24.8 \mathrm{kN} / \mathrm{m}^{3} \\ \text { Hysteretic damping factor }\left(\beta_{\mathrm{d}}\right) & =0.05\end{array}$

The impounded water is taken as inviscid, and compressible fluid with unit weight equal to $9.81 \mathrm{kN} / \mathrm{m}^{3}$, and pressure wave velocity $\mathrm{C}=1440 \mathrm{~m} / \mathrm{sec}$.

\section{Results}

The FE-(FE-HE) model is prepared based on the discretization and basic parameters explained in previous section. The responses of dam crest are initially obtained for this model by employing the previously efficient hyper-element [7] due to upstream, vertical and cross-stream excitations for one values of wave reflection coefficient $\alpha=0$. Then, these results in addition to case $\alpha=0.5$ are obtained utilizing the new proposed modified efficient hyper-element. It is noticeable that the results which are related to $\alpha=0.75$ could be found in [8].

It should be mentioned that response quantities which will be presented in this section are the amplitudes of the complex valued radial accelerations for two points located at dam crest (fig. 1). This is either the mid-crest point $\left(\theta=0^{\circ}\right)$ selected for upstream or vertical excitations or a point located at $\left(\theta=13.25^{\circ}\right)$ which is used for the case of cross-stream excitation. This is due to the fact that radial acceleration is zero at mid-crest for the cross-stream type of ground motion.

In each case, the amplitude of radial acceleration is plotted versus the dimensionless frequency for a significant range. The dimensionless frequency for upstream and vertical excitation is defined as $\omega / \omega_{1}^{S}$ where $\omega$ is the excitation frequency and $\omega_{1}^{S}$ is the fundamental frequency of the dam on rigid foundation with empty reservoir for a symmetric mode. For the cross-stream excitation cases, the dimensionless frequency is defined as $\omega / \omega_{1}^{a}$, where $\omega_{1}^{a}$ is the fundamental resonant frequency of the dam on rigid foundation with empty reservoir for an anti-symmetric mode.

As mentioned above, several values of $\alpha$ are considered and results are obtained for different types of excitations. Analyses are carried out based on the modified efficient method proposed herein and in each of these cases; the response is compared against the exact method. However, prior to this presentation, it is worthwhile to have a glance of a similar comparison for the original efficient method, which was obtained in the previous study [7]. This is 
provided in fig. 4 only for the worst case (i.e., maximum error in comparison to exact method) that was calculated for $\alpha=0$. Thereafter, the current study results for the modified efficient procedure are presented in figs. 5-6 for two $\alpha$ values mentioned above (i.e., $\alpha$ equal to 0.0 and 0.50 ). It is also worthwhile to mention that $\bar{\omega}=\omega_{1}^{S}$ is chosen for all these cases.
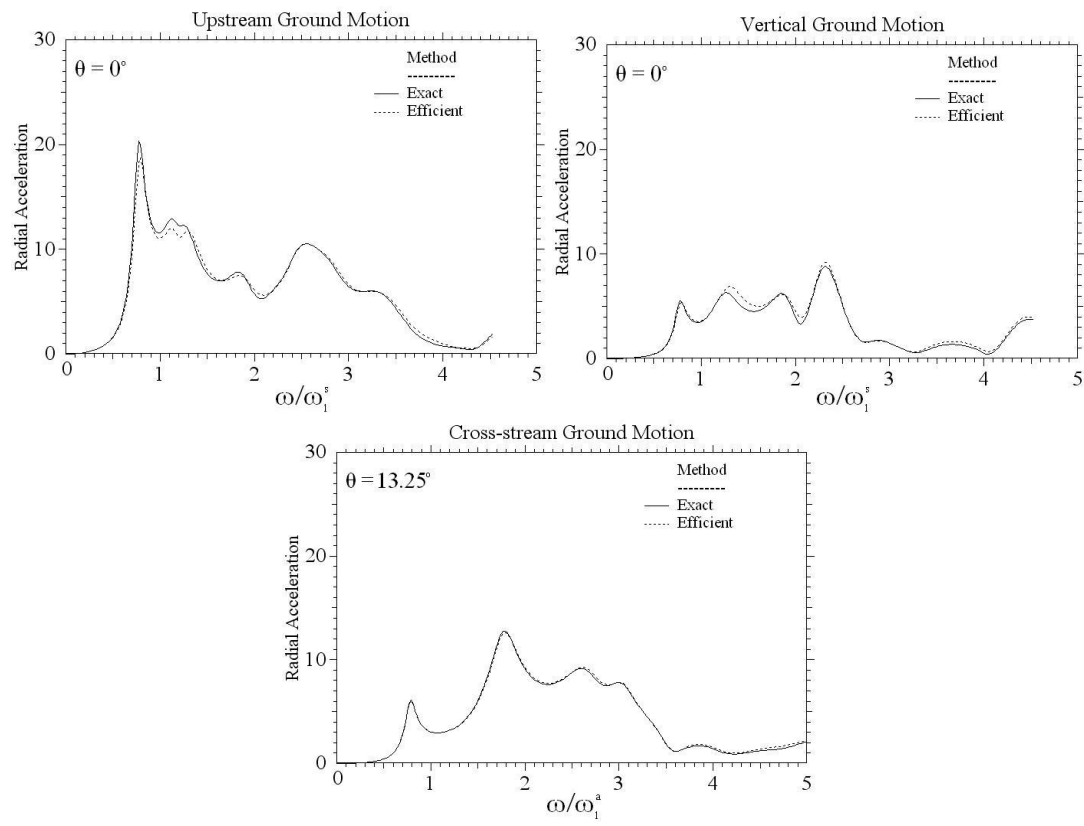

Figure 4: Responses due to different excitations $(\alpha=0)$ : Efficient method [7].

It should be noted that fig. 5 is directly comparable with fig. 4 , and one can capture a feeling of what degree of improvement is yielded for the modified efficient procedure in comparison to the original efficient method. However, to make a more quantitative comparison, the percentage error is also calculated at the frequency corresponding to the first major peak of the response for both these methods (under this worst condition case of $\alpha=0$ ), and it is presented in Table 1. It is observed that percentage error for the original efficient approach could reach up to about $10 \%$, which is occurring for the upstream excitation. However, this small amount of error is decreased even further to the maximum value of about $0.5 \%$ in the case of modified efficient procedure which is an incredible improvement. 
116 Fluid Structure Interaction and Moving Boundary Problems IV
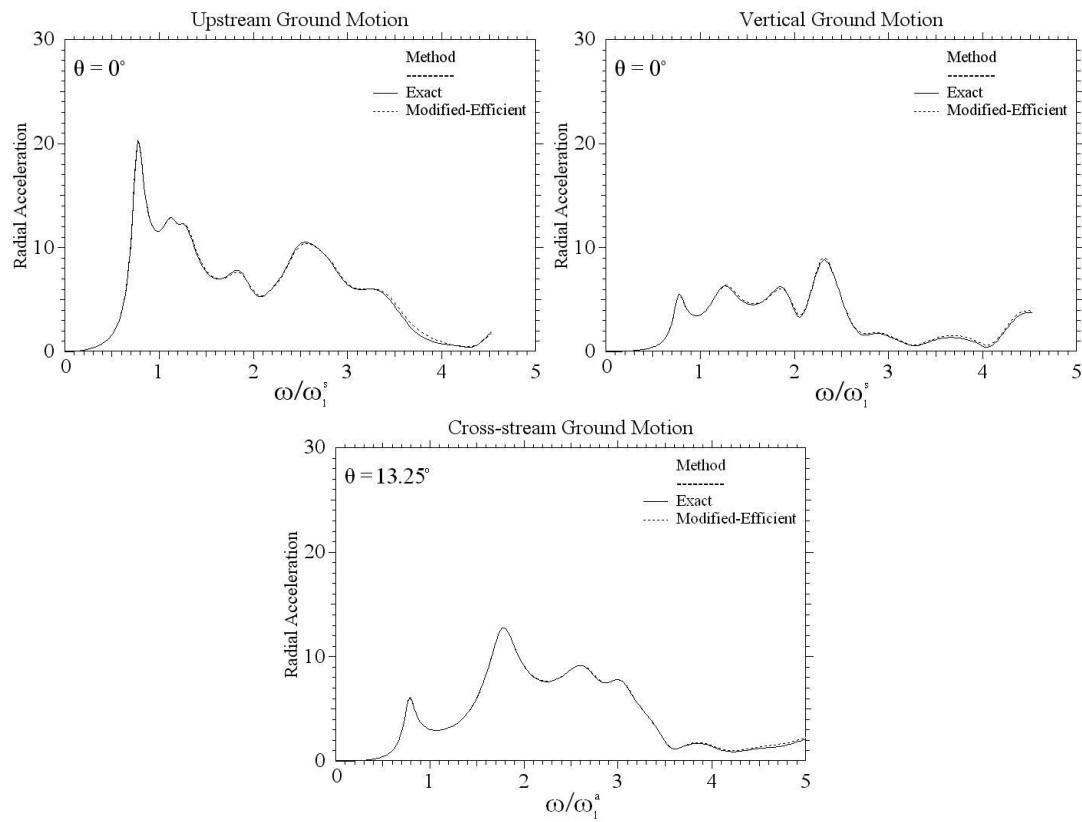

Figure 5: $\quad$ Response due to different excitations $(\alpha=0)$ : Modified-Efficient method.
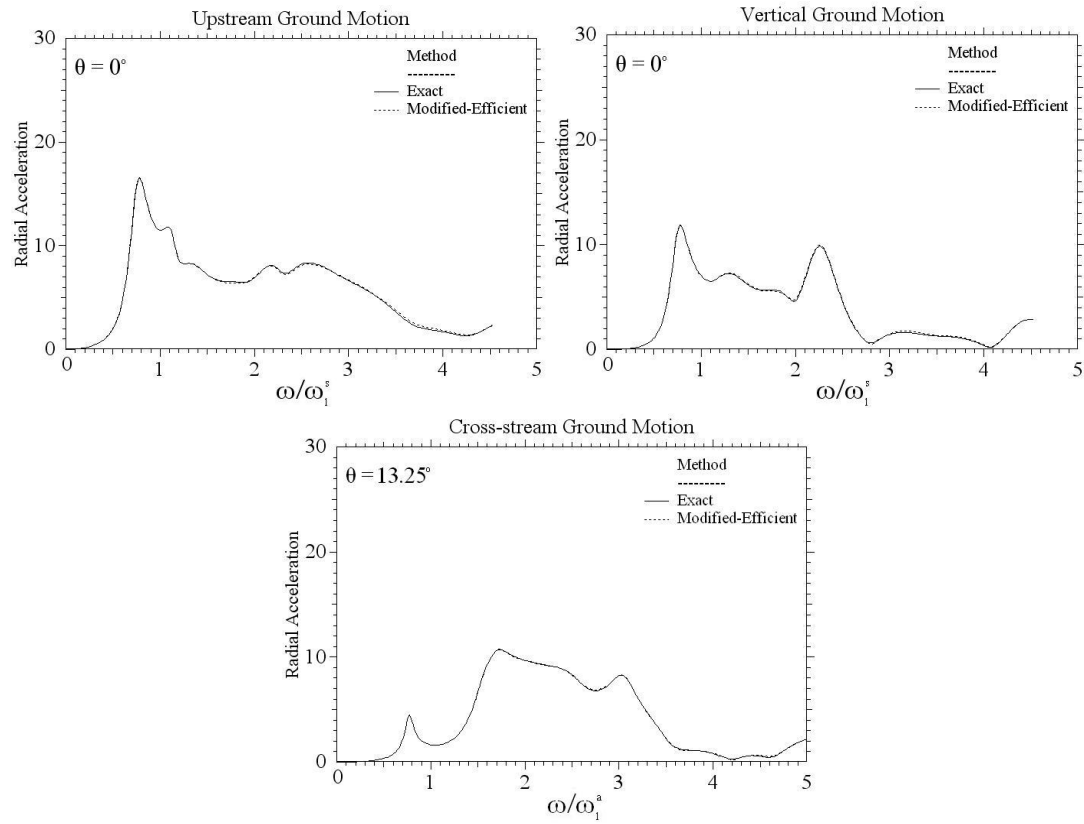

Figure 6: Responses due to different excitations $(\alpha=0.5)$ : ModifiedEfficient method. 
Table 1: Comparison of percentage error at the first major peak of response between the original efficient method and the modified efficient procedure for $\alpha=0$.

\begin{tabular}{|c|c|c|c||}
\hline Method & Upstream & Vertical & Cross-stream \\
\hline Efficient [7] & 9.79 & 5.03 & 1.65 \\
\hline Modified Efficient & 0.17 & 0.55 & 0.08 \\
\hline
\end{tabular}

Let us now concentrate purely on the results of the modified efficient method for different values of wave reflection coefficient $\alpha$ (figs 5 and 6). It is observed that response in each case matches very well with the exact response such that it is hardly distinguishable from the corresponding exact curve. Once again, it is worthwhile to quantify the approximation involved. For this purpose, the percentage errors for these cases are also calculated at the frequency corresponding to the first major peak of the response. These values are presented in Table 2. It is observed that percentage errors for all cases are very small, and the maximum error is about $0.5 \%$. This proves that the modified efficient method is a very robust technique and it is working extremely well under different conditions, some of which would be considered as highly challenging cases.

Table 2: $\quad$ Percentage error at the first major peak of response for the modified efficient method.

\begin{tabular}{||c|c|c|c||}
\hline$\alpha$ & Upstream & Vertical & Cross-stream \\
\hline 0.0 & 0.17 & 0.55 & 0.08 \\
\hline 0.5 & 0.54 & 0.18 & 0.23 \\
\hline
\end{tabular}

\section{Conclusions}

The formulation based on FE-(FE-HE) procedure for dynamic analysis of concrete arch dam-reservoir systems, was implemented. Also, a modified efficient procedure was employed for the calculation of the impedance matrix of the three dimensional fluid hyper-element. This was envisages to be an enhancement of a previously proposed efficient method [7]. A special purpose computer program was developed based on this methodology and the response of Morrow Point arch dam was studied as a typical example for various values of wave reflection coefficient $\alpha$, and different excitation types.

The results were always presented in comparison with the exact response so that one can easily capture a feeling of the possible error due to approximation introduced. Overall, the main conclusions obtained by the present study can be listed as follows:

- Initially, the percentage error at the first major peak of the response is calculated for the modified efficient procedure and it is compared against similar quantities obtained in a previous study for the original efficient 
method. This is carried out for $\alpha=0$ and three types of excitation. It is observed that percentage error for the original efficient approach could reach up to about $10 \%$, which is occurring for the upstream excitation. However, this small amount of error is decreased even further to the maximum value of about $0.5 \%$ in the case of modified efficient procedure which is an incredible improvement.

- In the next stage, the concentration was purely on the results of the modified efficient method for different values of wave reflection coefficient $\alpha$. Based on these results, it is observed that response in each case matches very well with the exact response such that it is hardly distinguishable from the corresponding exact curve. Percentage errors for these cases are also calculated at the frequency corresponding to the first major peak of the response. It is noted that percentage errors for all cases are very small, and the maximum error is about $0.5 \%$. This proves that the modified efficient procedure is a very robust and accurate method and it is working extremely well under different conditions, some of which would be considered as highly challenging cases.

- As mentioned before, the new approach is mainly relying on mode shapes corresponding to an arbitrary frequency. Thus, against the usual method which is depended on solution of complex eigenvalue problem for all excitation frequencies, the mentioned complex eigenvalue problem should be solved once for an arbitrary frequency in the new modified efficient procedure. It is obvious that this fact increases the analysis speed and procedure efficiency, significantly.

\section{References}

[1] Camara R.J., A method for coupled arch dam-foundation reservoir seismic behaviour analysis. Earthquake Engineering and Structural Dynamics, 29, pp. 441-460, 2000.

[2] Maity D. and Bhattacharyya S. K., Time-domain analysis of infinite reservoir by finite element method using a novel far-boundary condition. Finite Element in Analysis and Design, 32, pp. 85-96, 1999.

[3] Maeso, O., Aznarez, J.J. and Dominguez, J., Effects of space distribution of excitation on seismic response of arch dams. Engineering Mechanics Division, ASCE, 128(7), pp. 759-768, 2002.

[4] Lotfi, V., Direct frequency domain analysis of concrete arch dams based on FE-(FE-HE)-BE technique. Computers and Concrete, 1, pp. 285-302, 2004.

[5] Hall, J.F. and Chopra, A.K., Dynamic analysis of arch dams including hydrodynamic effects. Engineering Mechanics Division, ASCE, 109(1), pp. 149-163, 1983.

[6] Fok, K.-L. and Chopra, A.K., Frequency response functions for arch dams: hydrodynamic and foundation flexibility effects. Earthquake Engineering and Structural Dynamics, 14, pp. 769-795, 1986. 
[7] Lotfi, V., An efficient three-dimensional fluid hyper-element for dynamic analysis of concrete arch dams. Structural Engineering and Mechanics, 24(6), pp. 683-698, 2006.

[8] Aftabi Sani, A. and Lotfi, V., Linear dynamic analysis of arch dams utilizing modified efficient fluid hyper-element. Engineering Structures, Accepted for publication, 2007. 